\title{
Estimation des taux de recombinaison entre les locus Phi et Pgd et le complexe SLA chez le porc
}

\author{
G. GUERIN *, Christine RENARD ** et M. VAIMAN ** \\ avec la collaboration technique de Noëlle BOURGEAUX, P. DANDO, \\ Maryvonne JEGO et J.J. LEPLAT \\ *INRA, Laboratoire de Génétique biochimique, \\ Centre National de Recherches Zootechniques, F 78350 Jouy-en-Josas \\ ** Laboratoire de Radiologie appliquée, \\ INRA-CEA-IPSN-DPS, F 78350 Jouy-en-Josas
}

\begin{abstract}
Résumé
La méthode des lod scores a été utilisée pour tester les liaisons et estimer les taux de recombinaison $(\theta)$ entre les locus Phi et Pgd et le complexe SLA dans la descendance de croisements Piétrain $\times$ Large White et Large White $\times$ Large White. Nos résultats ne permettent pas de rejeter l'hypothèse d'indépendance entre les locus Phi et SLA et entre les locus Pgd et SLA. Les liaisons entre ces locus sont à exclure pour des taux de recombinaison respectivement inférieurs à 39 p. 100 et 36 p. 100 (rapport de vraisemblance de 0,01 ). Nos résultats tendent donc à infirmer l'hypothèse selon laquelle SLA serait situé entre les groupes Phi-H-Pgd et J-C. La liaison entre Phi et Pgd est confirmée avec un taux de recombinaison estimé de $\theta=8 \mathrm{p}, 100$. Les causes possibles des variations observées dans l'estimation des taux de recombinaison entre les locus Phi et Pgd sont discutées.
\end{abstract}

Mots clés : Porc, locus Phi, locus Pgd, complexe SLA, taux de recombinaison, histocompatibilité.

\section{Summary \\ Estimates of recombination rates between Phi and Pgd loci and the SLA complex in the pig}

The lod scores method was used to test linkage and to estimate recombination rates $(\theta)$ between Phi and Pgd loci and the SLA complex in the progeny of Piétrain $\times$ Large White and Large White $\times$ Large White matings. Our results do not allow rejecting the hypothesis of independence between Phi and SLA loci and between Pgd and SLA loci. Linkages between these loci were respectively excluded for recombination rates lower than 39 p. 100 and 36 p. 100 (likelihood ratio of 0.01). Our results thus would infirm the hypothesis of SLA being located between the Phi-H-Pgd and J-C groups. Linkage between Phi and Pgd is confirmed with an estimated recombination rate of $\theta=8 \mathrm{p}$. 100. Possible reasons for observed variations in the estimates of recombination rates between Phi and Pgd are discussed.

Key words : Pig, Phi locus, Pgd locus, SLA complex, recombination rate, histocompatibility. 


\section{Introduction}

Le taux de recombinaison entre les locus de groupes sanguins $\mathrm{C}$ et $\mathrm{J} \mathrm{du}$ porc a été initialement estimé à 5,3 p. 100 par ANDRESEN \& BAKER (1964) puis, en cumulant l'ensemble des données disponibles, à 5,75 p. 100 par MUIR \& RASMUSEN (1974). Le taux de recombinaison entre le locus J et le complexe majeur d'histocompatibilité du porc (SLA) varie selon les estimations de 9,82 p. 100 (HRUBAN et al., 1976) à 12,4 p. 100, celui entre les locus C et SLA étant de 16,4 p. 100 (HRADECKY et al., 1982). Ces résultats suggèrent l'ordre SLA-J-C (HRADECKY et al., 1982). Par ailleurs, RASMUSEN (1982) a estimé que les taux de recombinaison entre le groupe sanguin $\mathrm{H}$ et les locus $\mathrm{J}$ et $\mathrm{C}$ étaient respectivement de $41,7 \pm 3,5$ p. 100 et 42,4 $\pm 3,0$ p. 100, impliquant que les 2 segments portant $\mathrm{H}$ et SLA-J-C se situent sur le même chromosome. Or, le locus $\mathrm{H}$ est inclus dans un groupe de liaison contenant plusieurs gènes et notamment, de part et d'autre, le locus Phi de la phosphohexose isomérase et le locus Pgd de la 6-phosphogluconate déshydrogénase (ANDRESEN, 1971). Finalement, aucun de ces résultats ne permet de localiser le groupe de liaison Phi-H-Pgd par rapport au complexe SLA. Deux hypothèses sont envisageables : soit le complexe SLA est situé entre J-C et Phi-H-Pgd avec un taux de recombinaison entre Phi-H-Pgd et SLA de 25 p. 100 à 30 p. 100, soit le complexe SLA est situé à l'extérieur du segment reliant $\mathrm{J}$-C à Phi-H-Pgd (il serait alors génétiquement indépendant de ce dernier groupe). Vu l'intérêt de ces gènes et l'importance de leur localisation respective, nous avons cherché à estimer les taux de recombinaison entre les locus Phi, Pgd et le complexe SLA à partir de données familiales. De plus, cette étude nous a permis de réévaluer la distance entre les locus Phi et Pgd.

\section{Matériel et méthodes}

Deux groupes d'animaux provenant d'une lignée sélectionnée sur la prolificité au domaine expérimental d'Avord (Cher) ont été utilisés. Le premier était constitué des produits de croisement entre 6 verrats Piétrain et 81 truies de la lignée Large White, le second de familles issues de 8 verrats Large White et 24 truies de la lignée Large White, soit au total 893 produits typés pour les trois caractères SLA, PHI et PGD.

L'identification des caractères SLA a été réalisée avec une batterie de sérums utilisée par RENARD et al. (1982) selon la technique de TERASAKI \& MAC Clelland (1964) modifiée (RENARD et al., 1982) sur des lymphocytes préparés à partir de prélèvements de sang de moins de 24 heures. Le typage des caractères PHI et PGD a été effectué par électrophorèse sur gel d'amidon selon GuÉRIN et al. (1978) sur des échantillons conservés à $-20^{\circ} \mathrm{C}$ pendant moins de 3 mois.

La méthode des lod scores (MORTON, 1955) a été utilisée pour tester la liaison génétique et pour estimer les taux de recombinaison $\theta$ selon le maximum de vraisemblance, entre les locus pris deux à deux. Pour le couple de locus Phi et Pgd, le taux de recombinaison chez les mâles a été estimé sur des descendants issus de croisements où seul le parent mâle était double hétérozygote (fichier 1). Il a été procédé de même et réciproquement chez les femelles (fichier 2). L'écart entre les 
TAUX DE RECOMBINAISON CHEZ LE PORC

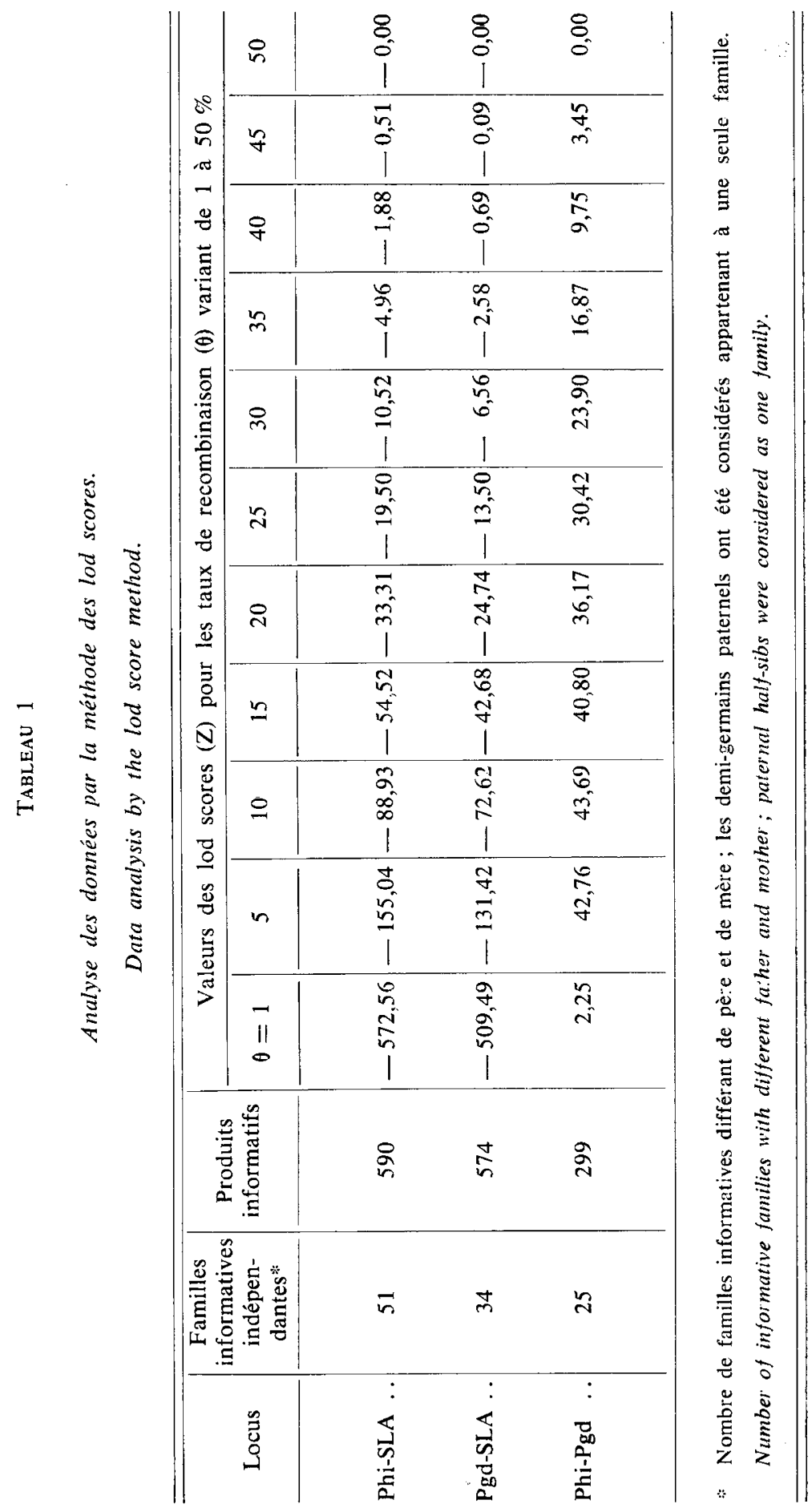


taux de recombinaison estimés chez les mâles et chez les femelles a été analysé par le test d'hétérogénéité selon CAVALLI-SFORzA \& BODMER (1971) d'après MorTON (1956), $\left(\Sigma \hat{Z}_{\mathrm{i}}-\hat{\mathrm{Z}}\right) \times 4,605$, qui suit approximativement la loi du $\chi^{2} \cdot \hat{Z}_{\mathrm{i}}$ représente la valeur maximum des sommes des valeurs des lod scores des pedigrees du fichier des mâles (fichier $1, i=1$ ) et du fichier des femelles (fichier $2, i=2$ ) et $\hat{Z}$ la valeur maximum de la somme des valeurs des lod scores des pedigrees de l'ensemble des 2 fichiers [fichier $(1+2)$ ]. Le programme de calcul selon la méthode des lod scores tient compte des phases parentales reconstituées par ascendance. Le complexe SLA a été assimilé à un seul locus : en effet, aucune recombinaison n'a été observée, dans cet échantillon, entre les 3 locus gouvernant les antigènes de classe I.

\section{Résultats}

Nos résultats ne permettent pas de rejeter l'hypothèse d'indépendance entre le locus Phi et le complexe SLA ni entre le locus Pgd et le complexe SLA. Les liaisons entre ces locus sont à exclure pour des taux de recombinaison respectivement inférieurs à 39 p. 100 et 36 p. 100 (lod score de $Z=-2$, soit un rapport de vraisemblance de 0,01) (tableaux 1 et 2). Par contre, la liaison entre les locus Phi et Pgd est confirmée avec un lod score maximum très élevé $(\mathrm{Zm}=44,01)$ pour une valeur de $\theta=8$ p. 100 . Cette valeur est de $\theta=4$ p. 100 chez les mâles et de $\theta=9$ p. 100 chez les femelles (tableau 3).

\section{TABLeaU 2}

Test des liaisons et estimation des taux de recombinaison entre les locus Phi et Pgd et le complexe SLA.

Test of linkages and estimates of recombination rates betweeen Phi and Pgd loci and the SLA complex.

\begin{tabular}{c|c|c|c|c|c|c}
\hline Locus & $\begin{array}{c}\text { Lod score } \\
\text { maximum } \\
(\mathrm{Zm})\end{array}$ & $\begin{array}{c}\text { Taux de re- } \\
\text { combinaison } \\
(\theta) \text { en } \%\end{array}$ & $\begin{array}{c}\text { Test de } \\
\text { liaison }\end{array}$ & $\begin{array}{c}\chi^{2} \\
\text { d'hétéro- } \\
\text { généité }\end{array}$ & $\begin{array}{c}\text { Degrés } \\
\text { de } \\
\text { liberté }\end{array}$ & $\begin{array}{c}\text { Test } \\
\text { d'hétérogénéité }\end{array}$ \\
\hline Phi-SLA ... & $-0,00$ & - & NS (a) & 48,83 & 50 & NS ; P $>0,10$ \\
Pgd-SLA ... & $-0,00$ & - & NS (b) & 36,70 & 33 & NS ; P $>0,10$ \\
Phi-Pgd ... & 44,01 & 8 & $* * *$ (c) & 43,94 & 24 & P $<0,01$ \\
\hline
\end{tabular}

(a) Borne inférieure de 39 p. 100 pour un rapport de vraisemblance de 0,01 $(Z=-2)$. Lower limit of 39 p. 100 for a likelihood ratio of $0.01 \quad(Z=-2)$.

(b) Borne inférieure de 36 p. 100 pour un rapport de vraisemblance de $0,01(\mathrm{Z}=-2)$. Lower limit of 36 p. 100 for a likelihood ratio of $0.01(Z=-2)$.

(c) Test de liaison très hautement significatif. Very highly significant linkage test. 
TABleaU 3

Estimation des pourcentages de recombinaison entre les locus Phi et Pgd chez les mâles et chez les femelles.

Estimates of recombination rates between Phi and Pgd loci in the males and in the females.

\begin{tabular}{|c|c|c|c|c|c|c|c|}
\hline Sexe & $\begin{array}{c}\text { Familles in- } \\
\text { formatives } \\
\text { indépen- } \\
\text { dantes* }\end{array}$ & $\begin{array}{l}\text { Produits } \\
\text { informatifs }\end{array}$ & $\begin{array}{l}\text { Lod scores } \\
\text { maximum } \\
(\mathrm{Zm})\end{array}$ & $\begin{array}{l}\text { Taux de } \\
\text { recombi- } \\
\text { naison } \\
(\theta) \text { en } \%\end{array}$ & $\begin{array}{c}\chi^{2} \\
\text { d'hétéro- } \\
\text { généité }\end{array}$ & $\begin{array}{c}\text { Degrés } \\
\text { de } \\
\text { liberté }\end{array}$ & $\begin{array}{l}\text { Test } \\
\text { d'hétérogénéité }\end{array}$ \\
\hline$\hat{\sigma}$ & 3 & 92 & 16,33 & 4 & 11,97 & 2 & $\mathrm{P}<0,01$ \\
\hline 우 & 22 & 153 & 19,72 & 9 & 23,43 & 21 & $\mathrm{NS} ; \mathrm{P}>0,10$ \\
\hline
\end{tabular}

* Nombre de familles informatives différant de père et de mère; les demi-germains paternels ont été considérés appartenant à une seule famille.

* Number of informative families with different father and mother; paternal half-sibs were considered as one family.

\section{Discussion}

Nos résultats sont donc en faveur d'une indépendance génétique des locus Phi-Pgd et du complexe SLA et excluent ainsi l'hypothèse selon laquelle le complexe SLA serait situé sur le segment reliant les groupes Phi-H-Pgd et J-C. Il est donc soit à l'extérieur de ce segment soit, avec $\mathrm{J}$ et $\mathrm{C}$, sur un chromosome distinct de celui portant Phi-H-Pgd. Les résultats de GefFrotin et al. (1984) indiquent que le complexe SLA est localisé au voisinage du milieu du bras long du chromosome $n^{0} 7$. Cette localisation a été confirmée par les équipes de RABIN et al. (1985) et par Gellin (communication personnelle) bien que la cartographie fine reste à définir. La liaison significative entre les locus J-C et $H$ rapportée par RASMUSSEN (1982) situerait le groupe Phi-H-Pgd sur le même chromosome que SLA. FrIES et al. (1983), Fries et al. (1984), Tikhonov et al. (1984) tendraient, quant à eux, à le localiser sur le chromosome $\mathrm{n}^{\circ}$ 15. Des résultats complémentaires sont, à l'évidence, nécessaires pour trancher entre ces 2 hypothèses.

La liaison étroite entre les locus Phi et Pgd est confirmée. Les taux de recombinaison entre ces locus $(\theta=8$ p. 100) estimés par la méthode des lod scores s'écarte notablement de notre précédente estimation de $\theta=0,56 \mathrm{p} .100$ sur un échantillon de porcs Piétrain (Guérin et al., 1983). Par contre, ils se rapprochent des valeurs observées par d'autres auteurs tels que ANDRESEN (1971), OISHI \& ĀBE (1979), RASMUSEN et al. (1980), JoRgenSEN (1981), NiELSEN (1983), HojNy et al. (1985), GAHNE $\&$ JUNEJA (1985) comprises entre $\theta=5,5$ p. 100 et $\theta=12,4$ p. 100 . De telles variations peuvent être dues à des différences de taux de recombinaison entre individus, comme l'ont déjà remarqué par exemple VAN ZEVEREN et al. (1985), GAHNE \& Juneja (1985) chez le porc et ANDERsson \& SANDBERg (1984) chez le cheval, et 
aussi au nombre peu élevé de parents doubles hétérozygotes utilisés. Ces différences peuvent expliquer l'hétérogénéité significative observée entre croisements dans l'estimation du taux de recombinaison entre les locus Phi et Pgd sur l'échantillon total (tableau 2). Par ailleurs, on note que l'écart entre les taux de recombinaison estimés chez les mâles et les femelles n'est pas significatif $\left(\chi^{2}{ }_{1}=2,11 ; P>0,05\right)$. Enfin, une hétérogénéité inter-croisements, observée chez les mâles et non pas chez les femelles, est à interpréter avec précaution dans la mesure où elle peut simplement provenir des effectifs parentaux différents dans les 2 groupes (tableau 3).

\section{Reçu le 16 septembre 1985. \\ Accepté le 27 janvier 1986.}

\section{Remerciements}

Nous remercions le $\mathrm{D}^{\mathrm{r}}$ Catherine Bonarti (INSERM, Paris) pour ses remarques pertinentes, et pour nous avoir fourni l'algorithme, $M^{\text {"le }}$ Sylvie PASSE (INRA, Jouy-en-Josas) pour avoir écrit le programme des lod scores et le $\mathrm{D}^{\mathrm{r}}$ L. Ollivier (INRA, Jouy-en-Josas) pour avoir participé à des discussions constructives.

\section{Références bibliographiques}

Andersson L., Sandberg K., 1984. Genetic linkage in the horse. II. Distribution of male recombination estimates and the influence of age, breed and sex on recombination frequency. Genetics, 106, 109-122.

ANDRESEN E., 1971. Linear sequence of the autosomal loci PHI, $\mathrm{H}$ and 6-PGD in pigs. Anim. Blood Groups biochem. Genet., 2, 119-120.

ANDResen E., BaKer L.N., 1964. The C blood group system in pigs and the detection and estimation of linkage between the $C$ and $J$ systems. Genetics, 49, 379-386.

Cavalli-Sforza L.L., Bodmer W.F., 1971. The genetics of Human Populations, 965 pp. Freeman W.H. and Co, San Francisco.

Fries R., Stranzinger G., Voegeli P., Dolf G., 1983. Provisional assignment of the G-blood group locus to chromosome $\mathrm{n}^{\circ} 15$ in swine. J. Hered., 74, 426-430.

Fries R., Rasmusen B.A., Jarrell V.L., Maurer R.R., 1984. Mapping of the gene for G blood group antigens to chromosome 15 in swine. Anim. Blood Groups biochem. Genet., 15, 251-258.

Gahne B., Juneja R.K., 1985. Predition of the halothane (Hal) genotypes of pigs by deducing Hal, Phi, Po2, Pgd haplotypes of parents and offspring : results from a largescale practice in Swedish breeds. Anim. Blood Groups biochem. Genet., 16, 265-283.

Geffrotin C., Popescu P.C., Cribiu E.P., Boscher J., Renard C., Chardon P., Vaiman M., 1984. Assignment of MHC in swine to chromosome 7 by in situ hybridization and serological typing. Ann. Génét., 27, 213-219.

Guérin G., Ollivier L., Sellier P., 1978. Déséquilibres de linkage entre les locus Hal (Hyperthermie maligne), PHI et 6-PGD dans deux lignées Piétrain. Ann. Génét. Sél. Anim., 10, 125-129.

Guérin G., Ollivier L., Sellier P., 1983. Etude du groupe de liaison Hal, Phi et Pgd chez le Porc : disposition relative des trois locus et estimation des taux de recombinaison. Génét. Sél. Evol., 15, 55-64. 
Hojny J., Cepica S., Hradecky J., Pazdera J., 1985. The use of marker loci for the determination of $\mathrm{Hal}$ genotypes. Anim. Blood Groups biochem. Genet., 16, suppl. 1, 95 (abst.).

Hradecky J., Hruban V., Pazdera J., Klaudy J., 1982. Map arrangement of the SLA chromosomal region and the $\mathbf{J}$ and $\mathbf{C}$ blood group loci in the pig. Anim. Blood Groups biochem. Genet., 13, 223-224.

Hruban V., Simon M., Hradecky J., Jilek F., 1976. Linkage of the pig main histocompatibility complex and the J blood group system. Tissue Antigens, 7, 267-271.

JoRGENSEN P.F., 1981. Blood types and other biochemical markers for stress-susceptibility and meat quality in pigs. In : Froystein T., Slinde E., Standal N. (ed.), Porcine stress and meat quality, 146-159, Agricultural Food Research Society, As, Norway.

Morton N.E., 1955. Sequential tests for the detection of linkage. Amer. J. Hum. Genet., 7, 277-318.

Morton N.E., 1956. The detection and estimation of linkage between the genes for elliptocytosis and the Rh blood type. Amer. J. Hum. Genet., 8, 80-96.

MUIR W.M., RaSMUSEn M.A., 1974. A combined estimate of recombination between the genes for $\mathrm{C}$ and $\mathrm{J}$ blood groups in pigs. Anim. Blood Groups biochem. Genet., 5, 133-135.

Nielsen P.B., 1983. Phi-H-Pgd systemerne hos dansk Landrace og dansk Yorkshire. Kongelige Veterinaer-og Landbohøjskole. Institut for sterilitetsforskning. ẢRSBERETNING, Annual report, 78-84.

Oishi T., Aвe T., 1979. Polymorphism of PHI, 6-PGD, PGM and ADA enzymes in porcine red cells. Bull. Nat. Inst. Anim. Ind., 35, 9-18.

Rabin M., Fries R., Singer D., Ruddle F.H., 1985. Assignment of the porcine major histocompatibility complex to chromosome 7 by in situ hybridization. Cytogenet. Cell. Genet., 39, 206-209.

RASMUSEN B.A., 1982. Linkage between genes at the $\mathbf{H}$ blood group locus and the loci for C and J blood groups in pigs. Anim. Blood Groups biochem. Genet., 13, 285-289.

Rasmusen B.A., Beece C.K., Christian L.L., 1980. Halothane sensitivity and linkage of genes for $\mathrm{H}$ red blood cell antigens, phosphohexose isomerase (PHI) and 6-phosphogluconate deshydrogenase (6-PGD) variants in pigs. Anim. Blood Groups biochem. Genet., 11, 93-107.

Renard C., Chardon P., Vaiman M., 1982. The pig histocompatibility system SLA : serological study on a group of antigenic specificities. Anim. Blood Groups biochem. Genet., 13, 161-177.

Terasaki P.I., Mac Clelland J.D., 1964. Microdroplet assay of human serum cytotoxins. Nature, 204, 998-1000.

Tikhonov V.N., Nikitin S.V., Gorelov I.G., Troshina A.I., Bobovich V.E., Astakhova N.M., 1984. Mapping of the loci of the $G$ and $H$ blood group systems on the chromosome 15 of domestic and wild pigs. Genetika, 20, 662-671.

Van Zeveren A., Van de Weghe A., Bouquet Y., 1985. Blood marker systems and their linkage with halothane sensitivity in Belgian Landrace pigs. Anim. Blood Groups biochem. Genet., 16, suppl. 1, 81 (abst.). 